\title{
Kuriositäten bei Einbänden
}

Die heterogene Struktur historisch gewachsener Sammlungen bringt es mit sich, dass sich allerlei Kuriositäten unter den aufbewahrten Objekten befinden. Das Vorliegen von Kuriosität bezieht sich dabei auf das methodisch und gattungsspezifisch Übliche der jeweiligen Zeit. Abweichungen von der Norm konnten ungewollt durch Fehler oder Unachtsamkeiten in der Verarbeitung zustande kommen. Häufig geschahen sie aber bewusst, um mit der Form eine bestimmte inhaltliche Botschaft zu vermitteln. Kuriositäten in Verbindung mit Einbänden sind dabei von besonderem Interesse, weil es sich um das handelt, was man als Erstes von einem Buch wahrnimmt.

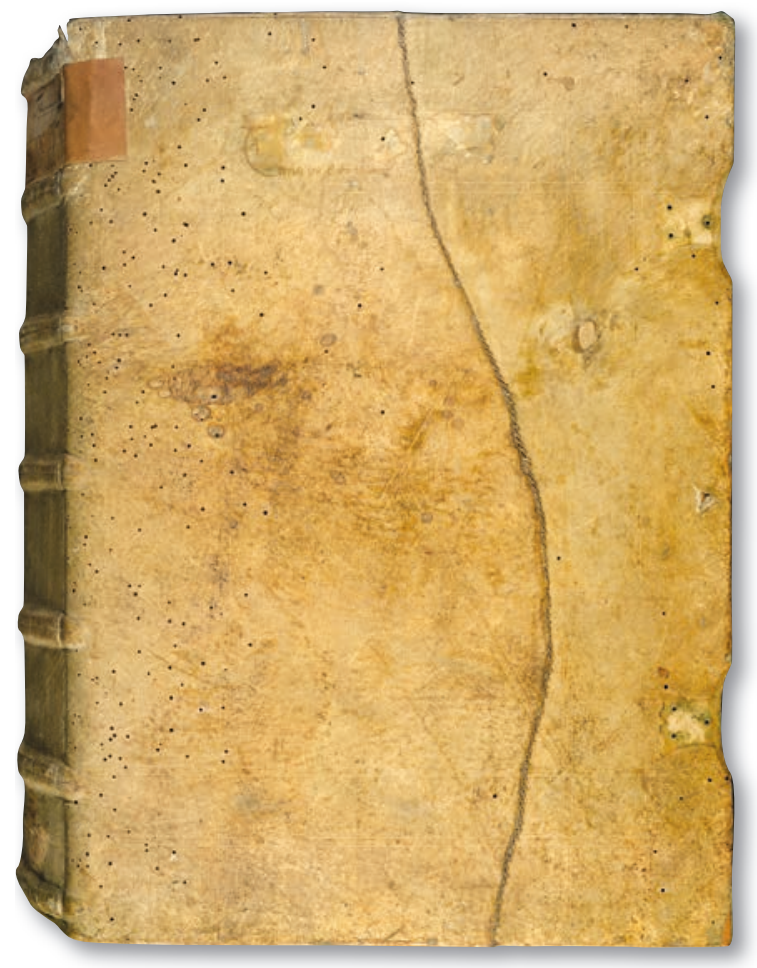

Abb. 1: Ledernaht (Inc.fol.1468)

\section{Buchbinderwerkstätten legten häufig eine auf} Sparsamkeit und effektive Nachnutzung vorhandener Materialien ausgerichtete Arbeitsweise an den Tag. So wurden Makulaturen nicht mehr benötigter Pergamenthandschriften, aber auch Fragmente von Inkunabeln, Fehl- und Probedrucke als Vorsatz, Falz- oder Bezugsmaterial des Einbands verwendet. Offenbar wurden aber auch Reste von Lederstücken benutzt. Bei der Kalkulation des Materialbedarfs für den Einband einer Basler
Inkunabel (Inc.fol.1468) hatte sich ein Buchbinder allerdings verschätzt oder minderwertiges Material herangezogen. Der Schweinslederbezug weist

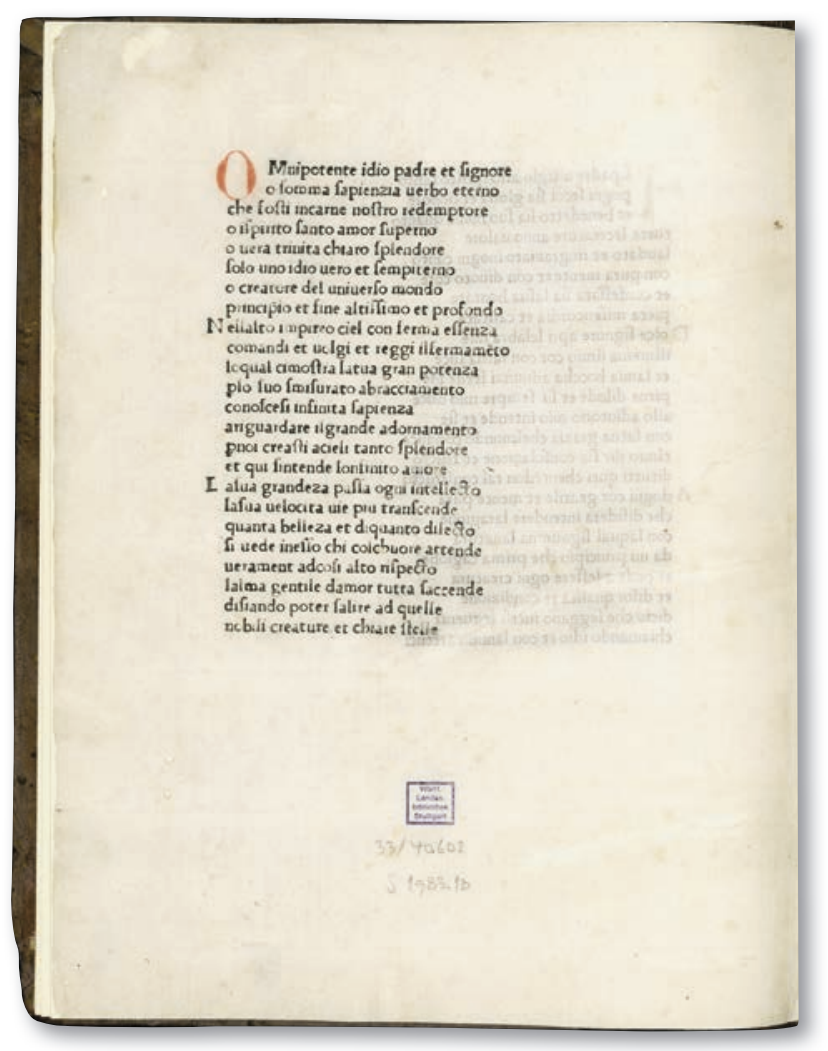

Abb. 2: Textseite und nachgenutzter Einband (Inc.fol.6028 b)

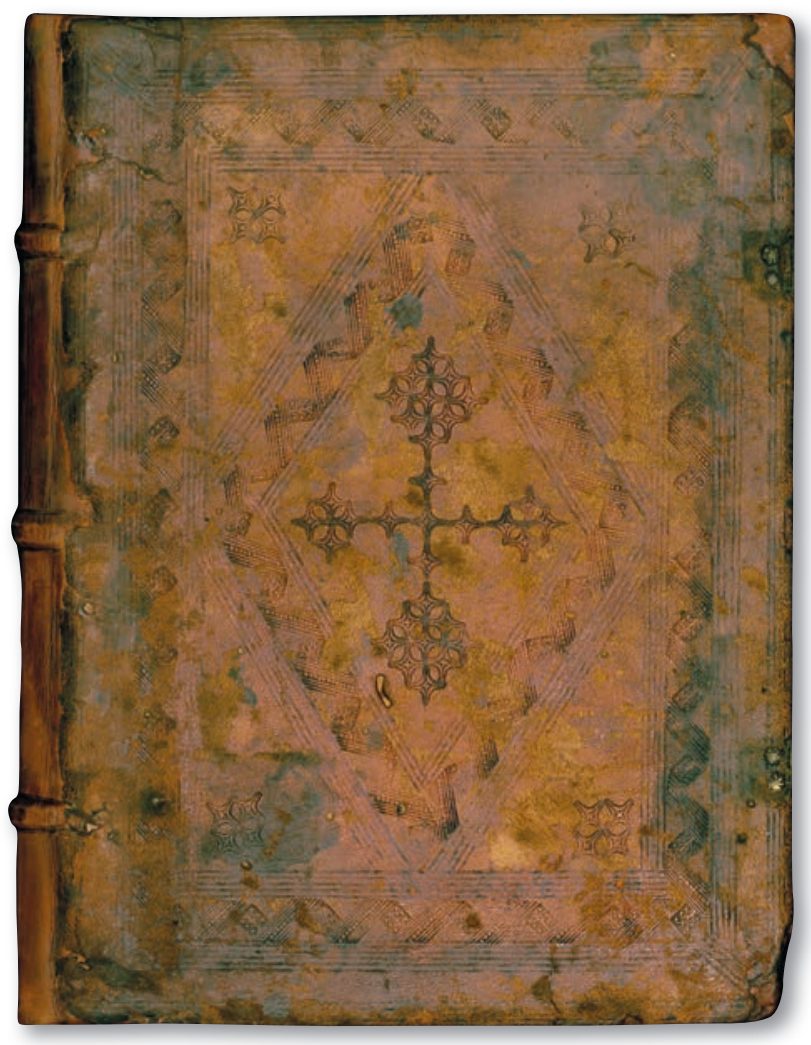


eine Ledernaht auf. Zwei Reststücke wurden beim Binden oder bereits im Vorfeld zu einem größeren Stück Leder verbunden. Solch ein Einband war für detailliertere Verzierungen nicht mehr geeignet und wurde daher nur mit Streicheisenlinien versehen.

Einbände wurden bis etwa zur Mitte des 19. Jahrhunderts individuell angefertigt und sekundär zum Buchblock hinzugefügt. Häufig kam es zu späteren Neubindungen, wenn der ursprüngliche Einband stark beschädigt war oder stilistisch nicht mehr dem Geschmack eines neuen Besitzers entsprach. Bei einer italienischen Inkunabel (GW 8015: Inc. fol.6028 b) erfolgte die Neubindung allerdings in anderer Weise. Die kleinformatige Schrift erhielt vermutlich im 18. oder frühen 19. Jahrhundert einen Einband nicht im zeitgenössischen Stil, sondern als Nachnutzung eines ursprünglich für eine großformatige, andere Inkunabel verwendeten gotischen Einbands. Es handelt sich um ein remboîtage genanntes Verfahren, weil zwei zuvor separat und in anderem Zusammenhang genutzte Teile des Buches vereint wurden. Darauf deutet auch ein aus dem vorderen Spiegel gelöstes Blatt
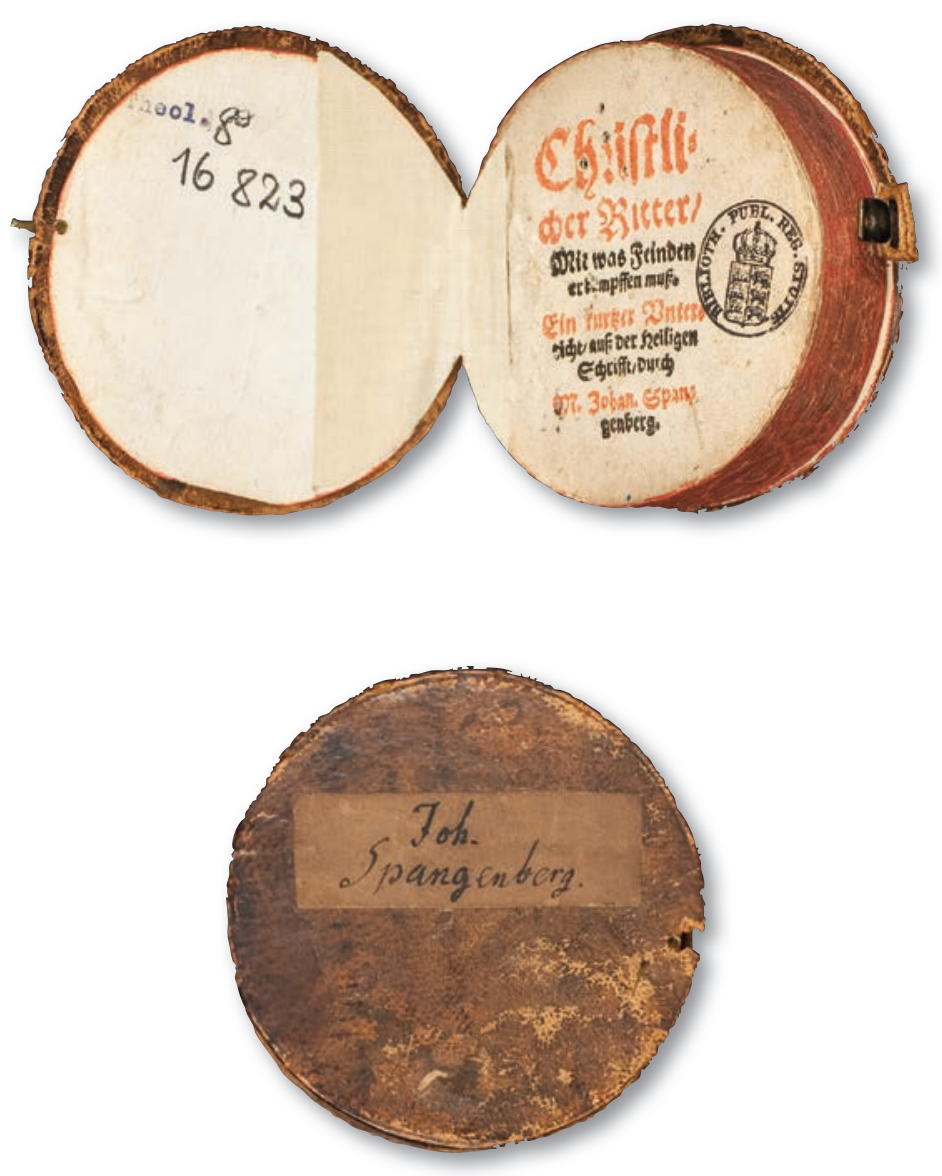

Abb. 3: Kreisförmiger Einband (ES 11)

1) Dazu Hohl, Werner: Das Pellegrino-Motiv; in: Einband-Forschung 8 (2001), S. 12-14. Dieses Muster erschien auch in einem Vorlagenbuch (1530) des italienischen Malers Francesco di Pellegrino († 1552) (Faksimile 1908: WLB: 39a/90055). mit Provenienzeinträgen des Jahres 1620 hin, das zu der ursprünglich mit diesem Einband versehenen Inkunabel gehörte.

Insbesondere bei christlicher Erbauungsliteratur wurden gelegentlich ungewöhnliche Materialien und Formate gewählt. Man wollte die kleinformatigen Bände dadurch in ihrem Erscheinungsbild hervorheben und die Notwendigkeit eines vorsichtigen Gebrauchs artikulieren. Es handelte sich um ursprünglich für häufige Verwendung bestimmte Literatur, deren Inhalt und Zweck jedoch dem Verschleiß standhalten sollte. So erhielt ein nicht exakt firmiertes, ca. 1550 gedrucktes Gebetbuch des lutherischen Theologen Johann Spangenberg (1484-1550) einen kreisförmigen Kalbsledereinband über Pappe (Theol.oct.16810/ES 11). Die Einbanddeckel der beiden Bände werden jeweils durch einen Gewebefalz als Gelenk und eine Schließe zusammengehalten.

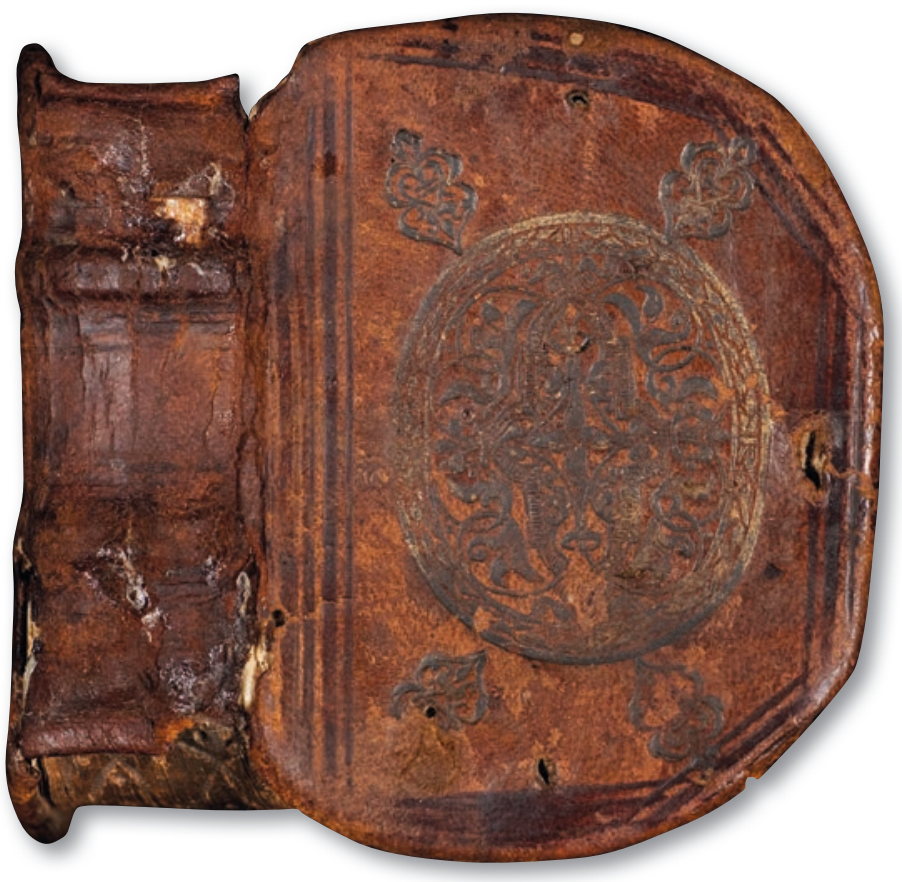

Abb. 4: Korbbogen-Einband (ES 16)

Aus dem Kloster Weingarten stammt ein katholisches Gebetbuch in lateinischer Sprache, Köln 1589 (HBF 2991/ES 16). Das Buch hat die Form eines Korbbogens. Der Kalbsledereinband wurde in der ca. 1560 bis 1600 tätigen Nürnberger Werkstatt Hans Pfisters (EBDB w003206) mit Streicheisenlinien und ornamentalen Motiven im Blindprägeverfahren verziert. Dabei fällt im Mittelfeld eine Platte mit Mauresken im Stil des sogenannten PellegrinoMotivs ins Auge. ${ }^{1}$ Vier Eckstempel mit Mauresken 
säumen das zentrale Motiv. Dass ein Kölner Druck in Nürnberg gebunden, dort wohl auch erworben wurde und schließlich in das Kloster Weingarten gelangte, deutet die Handelswege des damaligen Buchmarkts an.

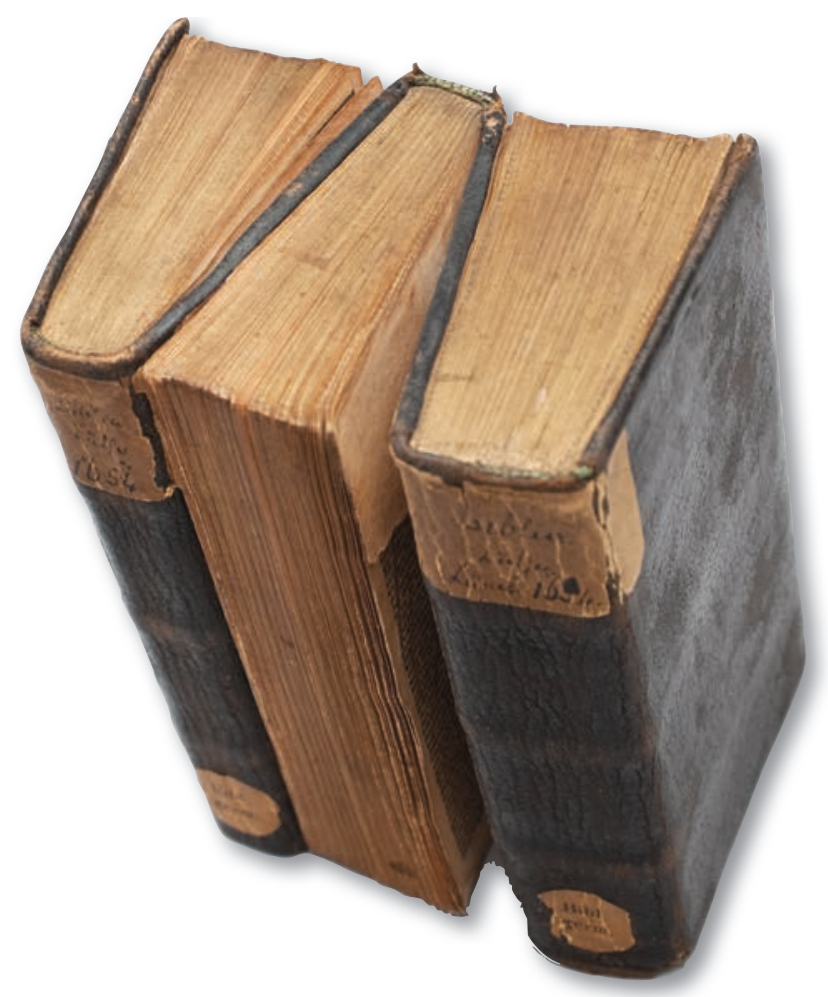

Abb. 5: Drillingsband (ES 37)

Aus der Sammlung Lorck kam eine Lüneburger Lutherbibel im Oktavformat (B deutsch 1654 01/ ES 37) in die Bibelsammlung. Die Freude der Barockkunst an Komplexität bei Ausstattung und Verzierung verband sich hier mit einer inhaltlichen Aussageintention. Diese Bibel wurde als Drillingsband gebunden, so dass der mittlere Teil jeweils einen Deckel mit den äußeren Teilen gemeinsam hat und zugleich seitenverkehrt eingebunden ist. Der mittlere Teil umfasst das Neue Testament, während die äußeren Teile zwei Hälften des Alten Testaments bieten. Als redender Einband deutet diese Komposition auf eine Akzentuierung des Neuen Testaments hin. Das Alte Testament läuft auf das Neue Testament als Mitte zu, allerdings nicht bruchlos - man muss das Buch drehen, um den mittleren Teil lesen zu können.

Ein theologisches Gefälle innerhalb des Neuen Testaments auf die Evangelien hin bringt der Drillingsband einer französischen Bibel (B franz.1738 02/ ES 38) zum Ausdruck. Der Mittelteil umfasst dabei neben den Evangelien auch eine Einführung in die

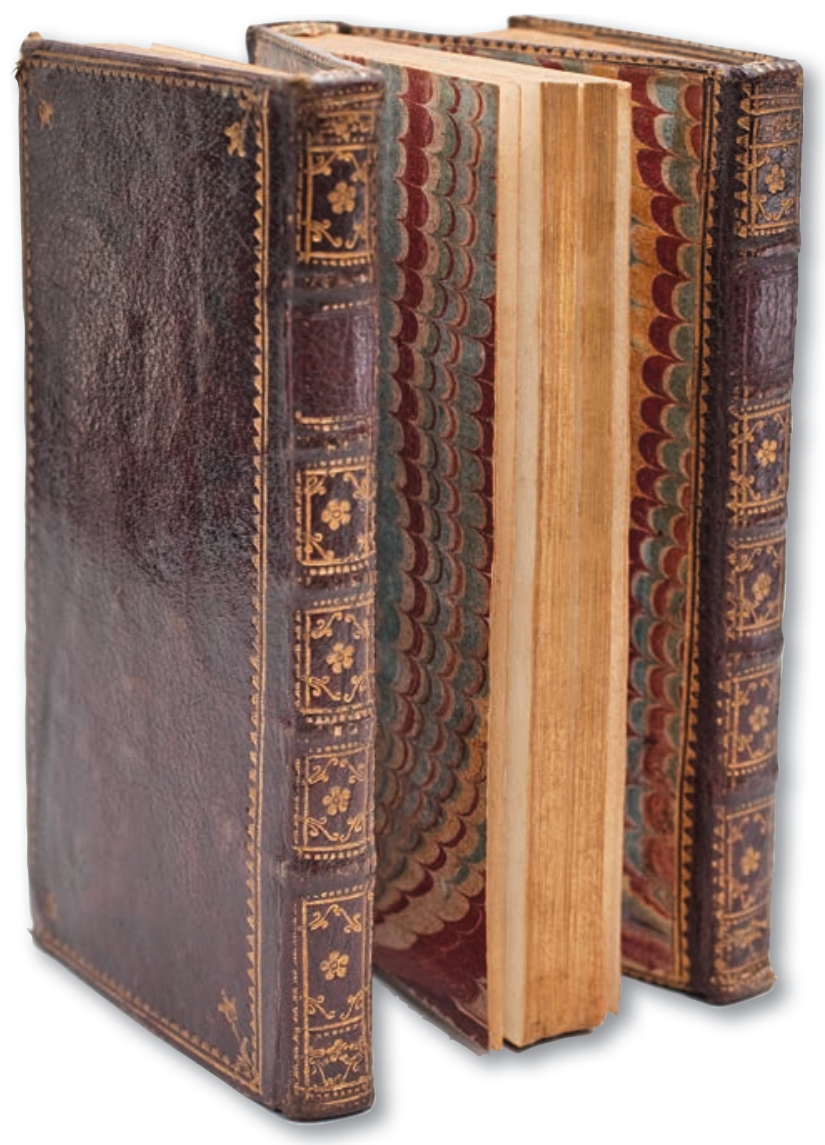

Abb. 6: Drillingsband (ES 38)

katholische Messe. So wird dem Leser verdeutlicht, dass der Gebrauch der Bibel vornehmlich in liturgischem Rahmen geschieht und die von den Evangelien bezeugte Gnade in sakramental-liturgischer Weise zuteilwird.

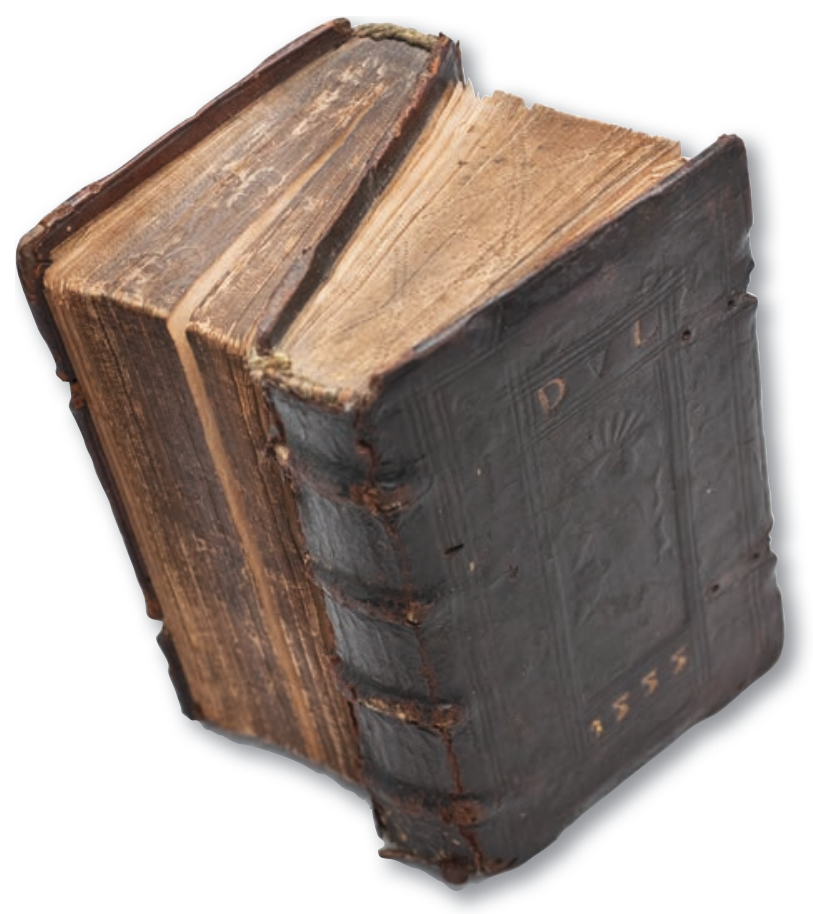

Abb. 7: Zwillingsband (ES 8) 
Der Rostocker Buchbinder Dietrich von Lohe († ca. 1590) erstellte für einen Sammelband von niederdeutschen, in Magdeburg gedruckten Schriften einen Zwillingseinband (B niederdt. 1553 03/ES 8). Die erste Buchhälfte umfasst die Übertragung von Luthers Übersetzung der Bücher Sirach bzw. Weisheit Salomos ins Niederdeutsche sowie anonym erschienene Gebete. Die zweite Hälfte enthält eine Sammlung der Kirchenlieder Luthers. Man könnte die Zusammenschau in der Einbandtechnik so interpretieren, dass rationale Lebensweisheit nicht von existenziell-emotionaler Frömmigkeit getrennt werden soll. ${ }^{2}$

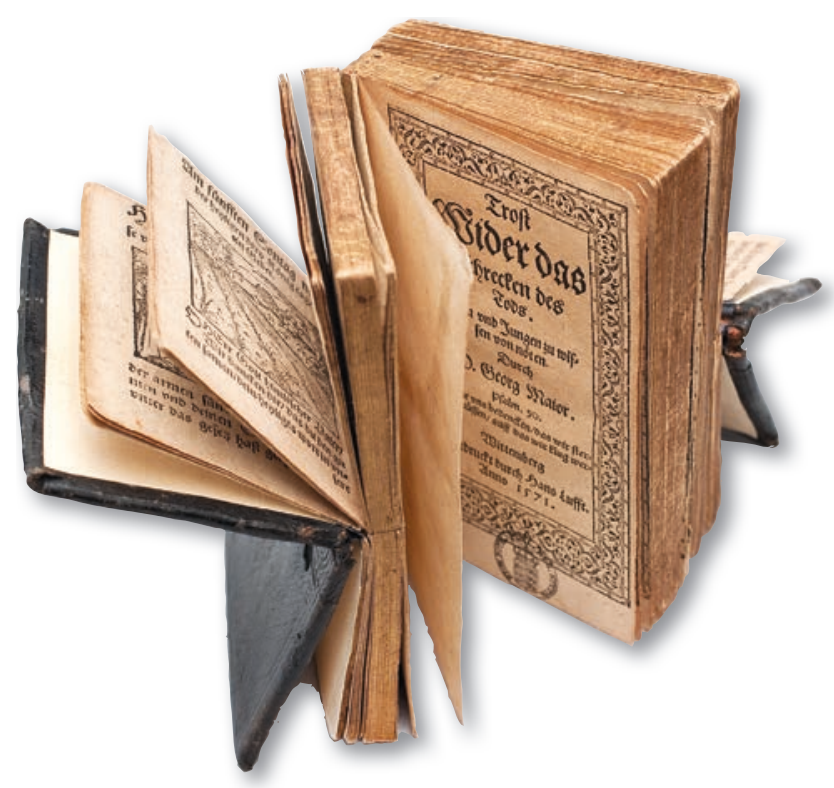

Abb. 8: Sechsfachband (ES 15)

Als Reisebibliothek im Miniaturformat und geistliche Wegzehrung sollte ein Sechsfachband mit überwiegend in Frankfurt/Oder gedruckten Werken des 16. Jahrhunderts dienen (HBF 2869/ES 15). Die äußeren Buchteile sind in sich geteilt und können in entgegengesetzte Richtung nach außen gekippt werden. Um alle sechs Kleinschriften mit Gebeten, Trostsprüchen und Texten zur Sterbebegleitung (ars moriendi) zu lesen, muss der Leser das Buch in alle Richtungen drehen bzw. aufklappen. Vielleicht wollte der Buchbinder neben der Freude an der buchtechnischen Kuriosität dadurch die Relevanz der Inhalte für alle Lebensphasen und -situationen zum Ausdruck bringen.

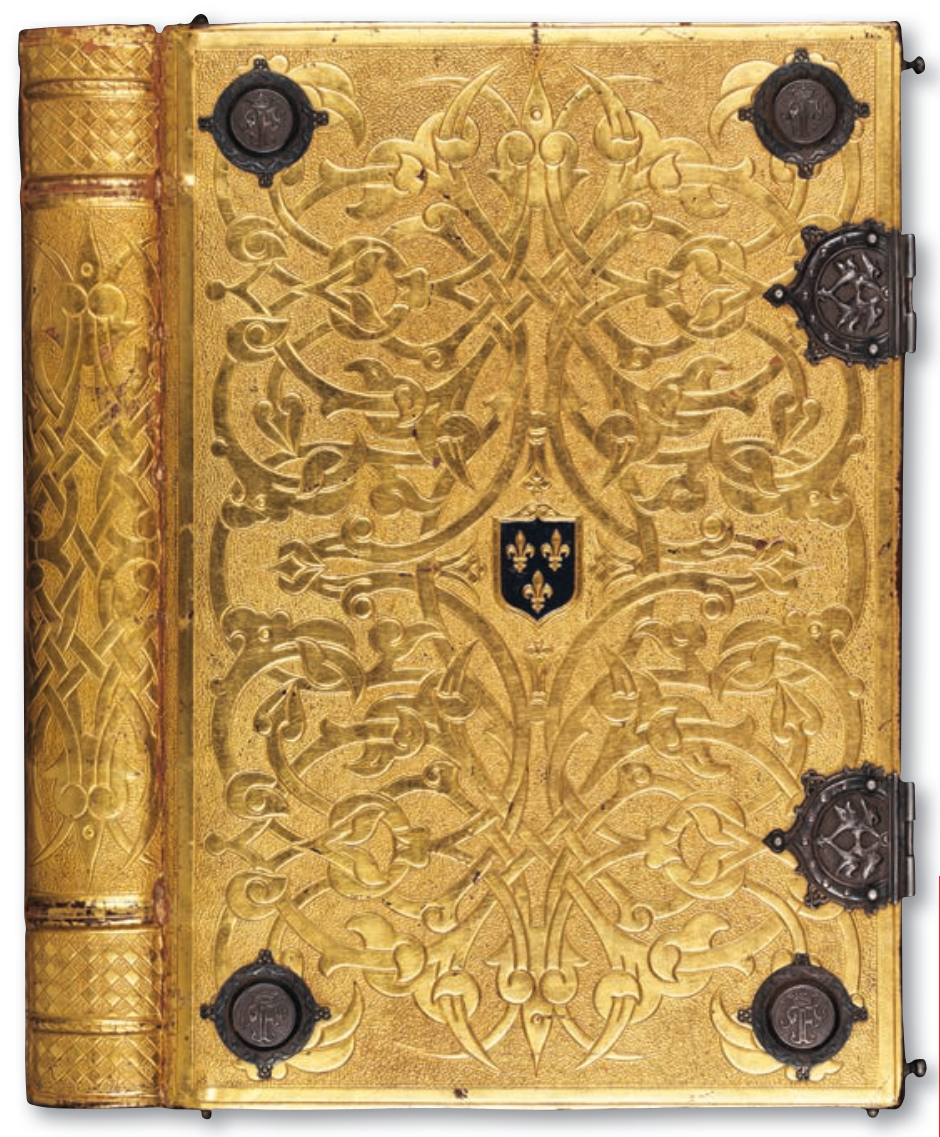

31

Abb. 9: Einband-Fälschung (ES IX)

Auch das Vortäuschen einer prominenten Provenienz mit Hilfe der Einbandverzierung ist ein kurioses Verfahren. So wurde eine Werkausgabe des italienischen Gräzisten Stephanus Niger (ca. 1475-1540) im 19. Jahrhundert neu gebunden (HBFa 792/ ES IX). Der Buchbinder Louis Hagué (1822-1891) ahmte in Goldschmiedetechnik die goldgeprägten Ledereinbände im Grolier-Stil des 16. Jahrhunderts nach. Charakteristisch ist dabei das vielfach in sich verschlungene Bandwerk mit filigraner Punktierung bzw. mit Schraffuren. Das zentrale Lilien-Wappen sowie das F-Monogramm auf den Metallbuckeln sollten auf den französischen König Franz I. (14941547) hindeuten. Die derart aufwendige Gestaltung des Einbands hätte durchaus im Auftrag dieses bibliophilen und kunstbegeisterten Königs geschehen können. Tatsächlich wollte jedoch ein über 300 Jahre später wirkender Buchkünstler seine Fähigkeiten unter Beweis stellen. Der gefälschte Einband gelangte 1963 als Teil der Sammlung des Engländers John Roland Abbey in die WLB. 